Article original

\title{
Evolution récente de la population, de l'occupation des sols et de la diversité floristique sur un terroir agricole du Sud-Ouest du Niger
}

\author{
Sanoussi Atta ${ }^{1 *}$, François AchARD² \& Sidi O.M. Ould Mohamedou 3 \\ ${ }^{1}$ Centre Régional AGRHYMET, BP 11011, Niamey, Niger; \\ IIRD, BP 11416, Niamey, Niger; \\ ${ }^{3}$ Commissariat à la Sécurité Alimentaire, Nouakchott, Mauritanie; \\ *Auteur pour les correspondances (E-mail : S.Atta@agrhymet.ne). \\ Reçu le 26-03-2009, accepté le 10-06-2010.
}

\begin{abstract}
Résumé
Au cours des 50 dernières années, un fort accroissement de la population a été enregistré dans la partie Sud-Ouest du Niger. Ce phénomène a entraîné de profonds changements dans l'occupation des sols et une pression accrue sur les ressources naturelles qui ont eu une incidence importante sur l'allure de la végétation. L'objectif de cette étude est de faire le point sur la richesse spécifique du terroir au regard de cette évolution. Les résultats montrent que la richesse floristique est assez élevée à Ticko. Ainsi, 400 espèces, réparties entre 66 familles ont été recensées. Parmi celles-ci, on compte 85 espèces ligneuses regroupées en 32 familles et 315 espèces herbacées, ou ligneuses basses, réparties entre 44 familles, dont $64 \%$ de Thérophytes. Cette flore représente $65 \%$ des espèces et $71 \%$ des familles présentes dans la région du Liptako. Après 25 ans de forte croissance démographique à Ticko, la diversité floristique reste encore à peu près intacte dans ce terroir. On note cependant une régression des populations d'espèces herbacées et ligneuses, autrefois bien représentées sur le terroir, qui pourrait conduire dans les années à venir à la disparition de ces taxons, et plus particulièrement d'une vingtaine de taxons ligneux.
\end{abstract}

Mots clés : population humaine, diversité floristique, Ticko, Niger.

\begin{abstract}
Population, Soil Occupation and floritical Diversity : Recent Evolution in a Southwestern Land in Niger
\end{abstract}

During the last 50 years, the population in south-west Niger (Liptako area) has grown rapidly. In Ticko, a village located in this area, the population has been multiplied by 7.5 between 1956 and 1996 to increase from 220 to 1 653 inhabitants. This population growth led to deep changes in land tenure, and accent pressure on that natural resources utilisation that have had an important impact on the vegetation patterns. This article aims at reviewing the current specific floristic diversity of the territory according to this evolution. In Ticko, the floristical diversity is quite high: 400 species, distributed over 66 families have been inventoried. Among them, 85 ligneous or phanerophytic species (32 families) and 315 herbaceous species or low ligneous species (chamephytic species) in 44 families. The therophytic species represent $64 \%$ of these latter. The flora represent $65 \%$ of the species and $71 \%$ of the botanical families found in the Liptako area $\left(30000 \mathrm{~km}^{2}\right)$.

In Ticko, the floristical diversity is quite intact after 25 years of rapid population growth. Nevertheless we observe a certain erosion in the herbaceous and ligneous populations which were important in diversity could lead, in the coming years, to the disappearance of these taxons, particularly of some 21 ligneous taxons. However this pessimistic observation must be tone down: on the other hand foreseeable disappearances are not certain; on the other hand a lot of new cultivated species are in the process of colonizing the village territory.

Key words: Niger, population growth, floristic diversity, Ticko. 


\section{Introduction}

Au cours des 50 dernières années, et particulièrement depuis le début des années 1970, une forte augmentation de la population a été enregistrée dans la partie sud de la région du Liptako, située au Sud-Ouest du fleuve Niger. C'est ainsi qu'au niveau du terroir de Ticko, la population est passée de 220 à 1653 habitants de 1956 à 1996, soit un taux de multiplication de 7,5. Quinze (15) chefs d'exploitation étrangers au village se sont installés à Ticko de 1956 à 1975, contre 74 de 1976 à 1996 (Achard et al., 1998). En 40 ans, la densité humaine, par rapport à la surface du terroir, est passé de 3 à 22 habitants. $\mathrm{km}^{-2}$, et par rapport à la surface agricole utile, de 6 à 41 habitants.km${ }^{2}$ (Bouzou, 1998). Comme dans toute l'Afrique de l'Ouest, cet accroissement de la population a été accompagné d'une augmentation du cheptel de bovins et petits ruminants (Winrock International, 1992) et d'une pression accrue sur les ressources naturelles avec d'importantes modifications au niveau de l'utilisation des terres. C'est ainsi que sur les plateaux, les peuplements ligneux et herbacés de la couverture arbustive contractée (brousse tigrée) ont connu une dégradation plus ou moins prononcée (Helmstetter, 1993 ; SeedCTFT, 1991 ; Millington et al., 1991). Sur les glacis, les défrichements se sont accélérés au détriment de la végétation naturelle et des jachères entraînant une fragmentation des parcours (Powell et Williams, 1995). Ce qui a eu pour conséquence la concentration d'un grand nombre de têtes de bétail sur des surfaces de plus en plus réduites pendant la saison des pluies. Cette surcharge a provoqué dans un premier temps une diminution de la production herbacée et, par la suite, des modifications dans la végétation des jachères et dans la succession végétale post-culturale (Hiernaux et Fernandez-Rivera, 1999 ; Hiernaux et Turner, 1996). Enfin, les bas-fonds, peu exploités jusqu'au début des années 1990, ont été presque entièrement aménagés pour des cultures de saison sèche depuis 1996.

La physionomie des formations végétales de cette région, et plus particulièrement celle des formations qui recouvraient les glacis, a été profondément modifiée. Ces modifications ontelles eu une incidence sur la diversité spécifique comme le remarquent Stuart et al. (1990)?

Dans le cadre du projet de recherches sur «l'amélioration et la gestion de la jachère en
Afrique de l'Ouest» une étude de la végétation des jachères et de sa dynamique interannuelle a été entreprise entre 1996 et 2001 sur le terroir de Ticko. Cette étude a été complétée par un inventaire de la végétation herbacée et ligneuse à l'échelle du terroir et par des enquêtes sur les formations végétales présentes dans les années cinquante. Parallèlement à l'inventaire floristique, des recherches concernant l'évolution, de 1956 à 1996, de la population et de l'occupation des sols ont été conduites. L'objectif de ce travail est de faire le point sur la biodiversité végétale du terroir, après 30 années de perturbation des formations originelles, et d'envisager son évolution dans les années à venir.

\section{Matériel et méthodes}

\subsection{Présentation du milieu d'étude}

Le terroir de Ticko (latitude $13^{\circ} 14^{\prime} \mathrm{N}$, longitude $1^{\circ}$ $\left.50^{\prime} \mathrm{E}\right)$, qui relève du canton de Torodi, est situé à $45 \mathrm{~km}$ au sud-ouest de Niamey sur la route Niamey-Ouagadougou (Figure 1). D'une superficie d'environ 7500 ha, il est constitué, comme tous les terroirs de la région, par trois unités géomorphologiques: les plateaux (2 702 hectares), les glacis sableux (4 $279 \mathrm{ha}$ ), les koris (cours d'eau à écoulement souvent violent mais éphémère) et les bas-fonds : kori de Séno Ticko et bas-fond du Diguibari (488 ha) (Banoin et Achard, 1998). Les plateaux sud et central délimitent un bassin versant (Séno Ticko) drainé par un kori qui se jette dans une rivière intermittente qui coule de juin à octobre appelée Diguibari, et qui limite le terroir au nord. Ce bassin versant est composé, pour partie, par de grands glacis en pente douce, défrichés et cultivés dans leur totalité depuis environ 25 ans seulement, car les points d'eau y sont rares et l'eau y est peu abondante. L'autre partie des terres cultivées est située sur le glacis qui s'étend entre la bordure nord du plateau central et le Diguibari, depuis le village de Kobadié, à l'Est, jusqu'à celui de Banguéniri, à l'Ouest.

Le terroir est soumis à un climat de type soudanosahélien, caractérisé par une longue saison sèche de novembre à avril suivie d'une période humide de mai à octobre. La pluviosité annuelle moyenne, enregistrée entre 1975 et 2000 , à Torodi situé à $12 \mathrm{~km}$ de Ticko, est de $580 \pm 57$ mm (Achard et al., 1999). 


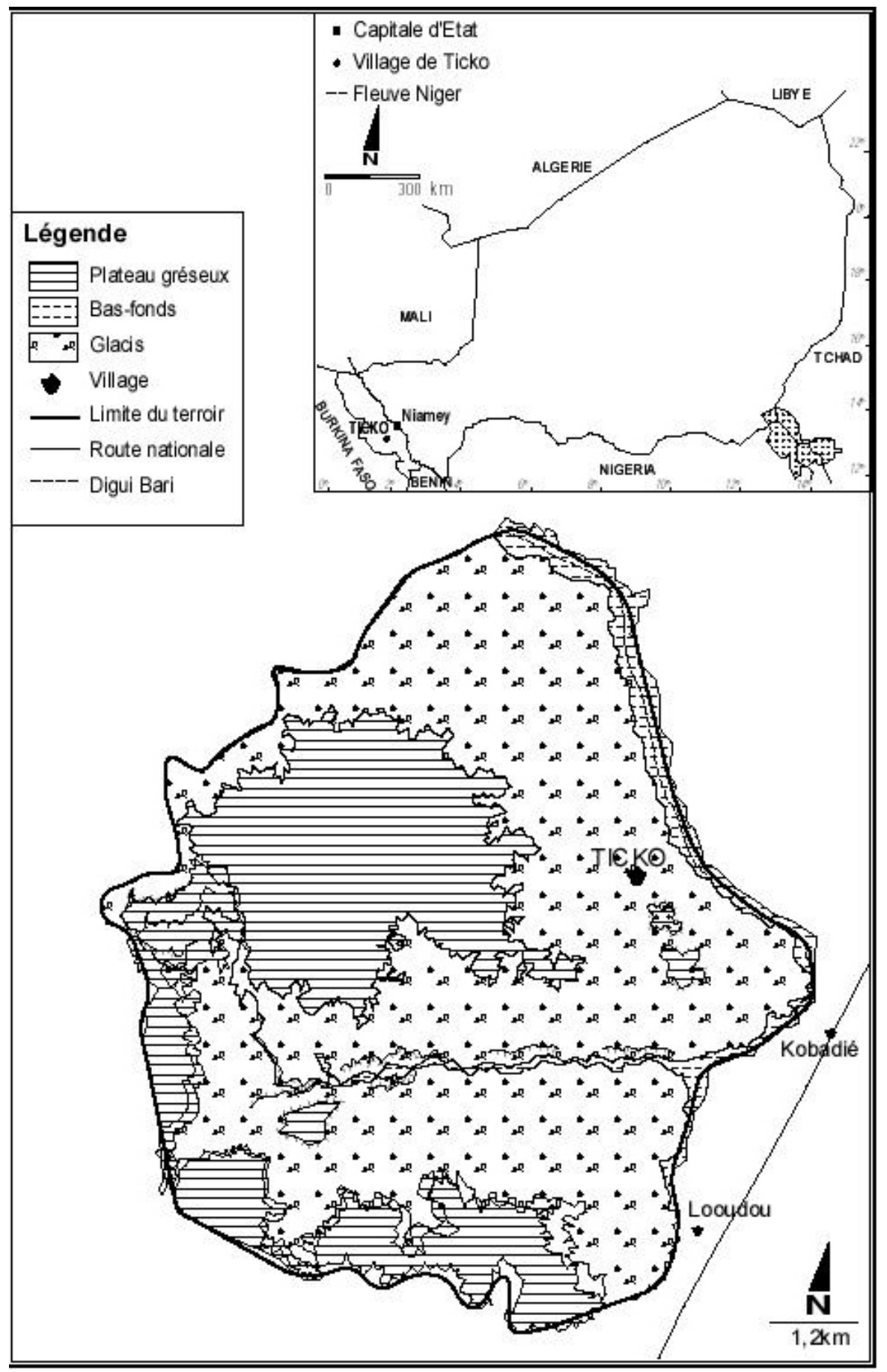

Figure 1: Carte géomorphologique de Ticko Source (Bouzou, 1998) 
Le sol du terroir est en général profond et supérieur à $2 \mathrm{~m}$ comme la plupart des sols de la zone cultivable du terroir située sur les glacis sableux. II appartient à la catégorie des «sols ferrugineux, peu lessivés, peu différenciés, sur sables pauvres en argile et limons» ( Gavaud et Boulet, 1967).

La population du Ticko est majoritairement constituée de Peuls venus du Fouta Toro, suivis des Zarmas. Un troisième groupe est constitué d'immigrants Haoussa venus suite aux sécheresses des années 1970 et 1984.

\subsection{Données disponibles}

Pour le terroir de Ticko, nous disposons de la carte topographique au 1/200 000, des photographies aériennes de 1956, 1975 et 1996 et d'une image SPOT du 03 Décembre 1996.

L'évolution de la population a aussi été estimée à partir des résultats des 2 recensements généraux de la population effectués en 1977 et 1988 (RGP, 1985 ; RGP, 1992). Ces données ont été complétées par des enquêtes auprès du poste administratif de Torodi et de la population locale, afin de préciser le nombre d'exploitations agricoles présent en 1956, 1975 et 1996, ainsi que les flux d'immigration et d'émigration à l'échelle du terroir.

\subsection{Interprétation des photographies aériennes}

Elle a consisté en la délimitation des différentes entités d'occupation des terres en fonction des signatures spectrales. Les discriminations ont porté sur :

- les couches des unités paysagiques et d'occupation des sols: les cultures, les jachères et friches, les plateaux, les talus, les bas-fonds, les jardins, les sols dégradés et les buttes;

- les routes et pistes;

- les villages.

\subsection{Numérisation}

La numérisation a été effectuée à l'aide d'une table à numériser. II s'agit de transformer la carte analogique en carte numérique. La digitalisation a été réalisée à l'aide du logiciel Cartalinx. Les fichiers issus de la digitalisation ont ensuite été exportés vers le logiciel ArcView qui a été utilisé pour les autres opérations de traitement jusqu'à la finalisation des cartes.
L'établissement de ces cartes a ainsi permis de mesurer l'évolution de l'occupation des sols au cours de ces trois périodes d'études (Ould Mouhamedou, 2001).

\subsection{Caractérisation des unités géomor- phologiques et de la végétation}

Compte tenu de l'homogénéité des états de surface au niveau du terroir, un seul transect a été réalisé pour caractériser le milieu. Ce transect a été orienté suivant la topographie, du bas-fonds vers le plateau correspondant à un gradient d'humidité et de profondeur du sol.

Pendant le parcours du transect, un comptage des ligneux a été réalisé au niveau de chaque unité paysagique au niveau de trois échantillons de $900 \mathrm{~m}^{2}$ chacun.

L'inventaire des plantes vasculaires (Angiospermes et Ptéridophytes) a été effectué à partir : (1) de la liste des espèces observées sur les jachères étudiées au cours de la première phase du projet (1996-2001), (2) du recensement, entre 1999 et 2001, de toutes les espèces rencontrées sur l'ensemble de la surface des 3 unités géomorphologiques. La détermination des espèces a été réalisée à l'aide des travaux de Aubréville (1950), Berhaut (1967), Geerling (1982), Hutchinson et Dalziel (1954, 1958, 1968, 1972), Le Bourgeois et Merlier (1995), Poilecot (1999) et Peyre de Fabrègues et Lebrun (1976).

\section{Résultats}

\subsection{Evolution de l'occupation des sols depuis 1956}

L'augmentation de la population au niveau du terroir de Ticko s'est traduite, comme partout au Niger par une augmentation concomitante des surfaces cultivées (Tableau 1). C'est ainsi que sur les glacis, ces surfaces qui étaient de 335 ha en 1956 sont passées à 3259 ha en 1996. L'augmentation des surfaces cultivées est particulièrement importante entre 1975 et 1996 où elles ont varié de 898 ha à 3259 ha, soit $80 \%$ de la superficie totale. L'accroissement des surfaces cultivées s'est effectué au détriment des jachères et des friches dont la superficie a connu une évolution inverse pendant cette période. En effet, leur superficie est passée de 3796 ha (92\% de la superficie totale) en 1956 à 657 ha (16\%) en 1996. A ces cultures s'ajoutent également les jardins dont les superficies sont passées de 20 ha en 1975 à 53 ha en 1996 (Tableau 1). 
Tableau 1: Evolution des superficies (en ha et en \%) de sol occupées de 1956 à 1996 sur le terroir de Ticko.

\begin{tabular}{|c|c|c|c|c|c|c|}
\hline \multirow[b]{2}{*}{ Occupation du sol } & \multicolumn{2}{|c|}{1956} & \multicolumn{2}{|c|}{1975} & \multicolumn{2}{|c|}{1996} \\
\hline & ha & $\%$ & ha & $\%$ & ha & $\%$ \\
\hline Cultures & 335,4 & 8,09 & 897,80 & 22,06 & 3259,50 & 80,64 \\
\hline Jachères et friches & 3796,3 & 91,52 & 3111,10 & 76,43 & 656,70 & 16,25 \\
\hline Sols dégradés & 16,3 & 0,39 & 41,10 & 1,01 & 73,20 & 1,81 \\
\hline Jardins & 0 & 0 & 20,00 & 0,50 & 52,60 & 1,30 \\
\hline Total & 4148 & 100 & 4070 & 100 & 4042 & 100 \\
\hline
\end{tabular}

\subsection{Composition de la végétation de Ticko}

\subsubsection{Richesse floristique}

L'identification des espèces végétales présentes sur le terroir de Ticko a permis de recenser 385 espèces réparties entre 66 familles. Douze familles (12) comptent 10 espèces ou plus et regroupent $66 \%$ du total des espèces recensées (Tableau 2) qui sont des Ptéridophytes. La strate arborée-arbustive compte 84 espèces et 31 familles, la strate herbacée (+ chaméphytes ligneux) 301 espèces et 44 familles.

La richesse floristique est plus élevée sur les plateaux (243 espèces) que sur les glacis et les bas-fonds, où elle est équivalente, respectivement 183 et 184 espèces (Tableau 3). Les espèces caractéristiques sont constituées par celles observées uniquement dans un milieu donné. Le pourcentage de ces espèces, qui varie suivant les milieux, est plus important au niveau des herbacées comparé aux ligneux (Tableau 3).

Le spectre biologique est dominé par les Térophytes et les Phanérophytes qui représentent plus de $80 \%$ des espèces (Tableau 3 ) dans les 3 milieux ( $91 \%$ sur les glacis). La proportion relative des ces deux groupes varie peu d'un milieu à l'autre et fait bien ressortir le caractère d'aridité du terroir de Ticko, même dans le basfond, où ne subsistent que quelques flaques d'eau de novembre à mars.

Tableau 2 : Principales familles botaniques du terroir de Ticko

\begin{tabular}{lccc}
\hline \multirow{2}{*}{ Familles } & \multicolumn{3}{c}{ Nombre d'espèces inventoriées } \\
\cline { 2 - 4 } Poaceae & 69 & 0 & Espèces \\
Fabaceae & 46 & 2 & $\mathbf{6 9}$ \\
Cypéraceae & 25 & 0 & $\mathbf{4 8}$ \\
Convolvulaceae & 16 & 0 & $\mathbf{2 5}$ \\
Mimosaceae & 0 & 15 & $\mathbf{1 6}$ \\
Rubiacées & 6 & 5 & $\mathbf{1 5}$ \\
Euphorbiacées & 8 & 5 & $\mathbf{1 1}$ \\
Malvaceae & 13 & 0 & $\mathbf{1 3}$ \\
Amarantaceae & 12 & 0 & $\mathbf{1 3}$ \\
Caesalpiniaceae & 6 & 5 & $\mathbf{1 2}$ \\
Acanthaceae & 10 & 0 & $\mathbf{1 1}$ \\
Capparidaceae & 4 & 6 & $\mathbf{1 0}$ \\
\hline \multicolumn{1}{c}{ Total } & $\mathbf{2 1 5}$ & $\mathbf{3 8}$ & $\mathbf{2 5 3}$ \\
\hline
\end{tabular}


Tableau 3 : Répartition des espèces dans les différents milieux.

\begin{tabular}{lccc}
\hline Type d'espèces & \multicolumn{3}{c}{ Milieux } \\
\cline { 2 - 4 } & Glacis & Plateaux & Bas-fonds \\
\hline Herbacées & $132\left(33^{\star}\right)$ & $190(68)$ & $139(63)$ \\
Ligneux & $51(6)$ & $53(8)$ & $45(17)$ \\
\hline Total & $\mathbf{1 8 3}$ & $\mathbf{2 4 3}$ & $\mathbf{1 8 4}$ \\
\hline
\end{tabular}

* : \% d'espèces inféodées au milieu

\subsubsection{Grands traits de la végétation}

\subsubsection{Végétation des plateaux}

Trois unités paysagères peuvent être individualisées sur les plateaux : la surface sèche du plateau, les milieux humides répartis sur cette surface, les versants qui relient les plateaux aux glacis(Figure 2).

Les plateaux portent une végétation arbustive arborée très variée malgré une exploitation de la forêt assez intense à la fin des années quatre vingts. Cette végétation est en majeure partie constituée par des arbustes de la famille des Combrétaceae : Combretum nigricans, C. glutinosum, C. micranthum et Guiera senegalensis, au sein desquels croissent tout un cortège d'espèces ligneuses secondaires. Parmi les 53 espèces identifiées, on rencontre des arbustes tels que Gardenia sokotensis, des lianes : Gymnema sylvestre, Strophantus sarmentosus, Cissus quadrangularis, etc. et des arbres comme Sclerocarya birrea, Bombax costatum, Adansonia digitata, Cassia sieberiana, Lannea acida, Stereospermum kunthianum, Albizzia chevalieri, Pterocarpus erinaceus, Boscia angustifolia, etc. Ces 2 derniers, très recherchés pour leur production fourragère en fin de saison sèche, sont surexploités et en danger. Enfin, on trouve des taxons peu fréquents sur ce milieu, tels que Lonchocarpus laxiflorus, Maerua crassifolia, Anogeissus leiocarpus, Tamarindus indica, Prosopis africana, Ximenia americana (Achard, 2001).

Les Combrétaceae, Capparidaceae et Rubiaceae qui forment la base de la végétation des plateaux ont souvent une régénération abondante et vigoureuse (Aboubacar, 2000) qui s'effectue par multiplication végétative (Saley, 2001) ou par semis. En revanche, la plupart des autres espèces ligneuses ne comptent que des individus âgés, avec un faible taux de régénération.
Trois grandes unités de végétation herbacée peuvent être individualisées hors des sous-bois. Deux sont dominées par des Poaceae annuelles : I'une, à Loudetia togoensis, est située sur les parties ouest et nord-ouest du plateau central, l'autre, à Microchloa indica recouvre les plages de décantation, en amont des bandes boisées, la troisième est constituée par une Poaceae pérenne naine : Tripogon minimus qui colonise les micro-cuvettes. La légumineuse Zornia glochidiata est omni-présente. Des populations très denses mais de faible surface de Sida cordifolia, marquent les anciens parcs à bétail.

Contrairement à ce que l'on peut observer sur les glacis, les milieux humides ne sont pas rares sur les plateaux. Ce sont pour la plupart des flaques d'eau éphémères de surface restreinte (quelques $\mathrm{m}^{2}$ à quelques dizaines de $\mathrm{m}^{2}$ ) dans des dépressions naturelles peu profondes (10 à 50 $\mathrm{cm})$. Il existe cependant 3 ou 4 mares plus importantes, s'asséchant en novembre seulement. Les abords de ces points d'eau, humides mais quelquefois inondés, sont recouverts d'espèces caractéristiques de ces milieux, hélophytes ou hygrophytes. On y observe des Poaceae telles que Echinochloa colonna, Eragrostis pilosa, Rytachne triaristata, Panicum laetum, Elytrophorus spicatus, des Cypéracées : Cyperus podocarpus, C. submicrolepis, Schoenoplectus senegalensis, Kyllinga tenuifolia, Mariscus squarosus, Eleocharis atropurpurea, des Astéraceae : Aspilia bussei, Bidens pilosa, Tridax procumbens, Vicoa leptoclada, ..., accompagnées par 2 légumineuses Aeschynomene tambacoudensis et Desmodium hirtum. Le plan d'eau lui même abrite des hydrophytes : une Pontédériaceae à fleur blanche : Heteranthera callifolia, une Alismataceae : Lophotocarpus guayanensis, une Fabaceae: Aeschynomene indica, une Poaceae vivace : Echinochloa stagnina, qui ici a un comportement d'annuelle. 


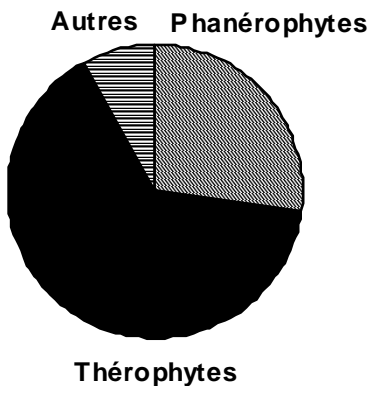

(A)

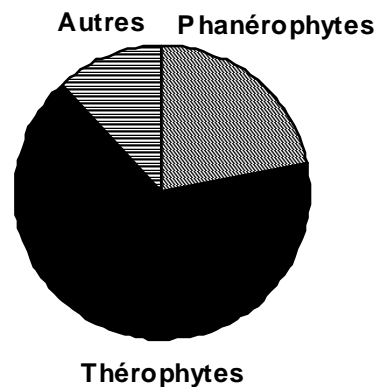

(B)

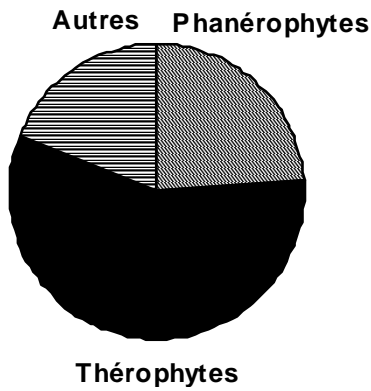

(C)

Figure 2 : Spectre biologique des espèces sur les glacis (A), les plateaux (B) et dans les bas-fonds (C).

\subsubsection{Végétation des glacis}

Le glacis du Diguibari a été le premier à être habité et est le plus peuplé actuellement. La végétation, qui le recouvrait il y a 50 ans, a pratiquement disparu. La surface en jachères y est faible, et ces dernières, surpâturées, sont recouvertes par des espèces annuelles à cycle court : Zornia glochidiata, Brachiaria xantholeuca et Mitracarpus villosus, dont la contribution spécifique atteignait, en 1999, 75\% à 94\% du total de la végétation (Achard et al., 1999). Seuls quelques arbres, principalement Balanites aegyptiaca et Combretum glutinosum, subsistent. La strate arbustive, lâche, est composée de Guiera senegalensis. Sur la partie basse du glacis, proche du Diguibari, on note quelques pieds de Tamarindus indica et d'Anogeissus leiocarpus.

La végétation des glacis de Séno Ticko est moins dégradée. On y observe un peuplement arboré relique, peu dense mais assez diversifié et $80 \%$ des jachères du terroir. Bien que la végétation de la majorité des jachères soit semblable à celle observée sur le glacis du Diguibari, on y trouve encore, sur des surfaces restreintes (1) des jachères de plus de 5 ans avec une grande Poaceae vivace: Andropogon gayanus, pâturée toute l'année et donc incapable de produire des semences, et des Poaceae annuelles à cycle long telles que Diheteropogon hagerupii et/ou Ctenium elegans rencopntrées, (2) des jachères anciennes (10 à 30 ans) à Loudetia togoensis et Zornia glochidiata dans la partie haute du glacis et (3), çà et là des jachères récentes à Pennisetum pedicellatum et Eragrostis tremula.
La végétation actuelle du glacis de Séno Ticko permet d'avoir une assez bonne idée de la végétation qui recouvrait le terroir dans les années cinquante, constituée par une forêt claire ou une savane boisée (Trochain, 1957) formée par :

- une strate herbacée à base de Poaceae vivaces: Andropogon gayanus, ou Poaceae annuelles à cycle long : Diheteropogon hagerupii, Andropogon fastigiatus $^{\star 1}$, A. pseudapricus*, Ctenium elegans et Pennisetum pedicellatum,

- une strate arbustive à base de Combrétaceae : Guiera senegalensis, Combretum micranthum, C. aculeatum, de Rubiaceae : Gardenia ternifolia, Feretia apodanthera*, de Capparidaceae : Boscia angustifolia, Maerua angolensis*, Cadaba farinosa*,

- un peuplement arboré clair avec Terminalia avicennoides*, Prosopis africana*, Combretum gluitnosum, Stereospermum kunthianum*, Bombax costatum*, Sclerocarya birrea, Piliostigma reticulatum, Albizzia chevalieri*, Entada africana*, Acacia nilotica, A. senegal.

Enfin, on note une population de palmiers doum (Hyphaene thebaica), en aval du village de Ticko, qui serait d'implantation assez récente (40 à 50 ans). II en est de même des Acacia albida, rares sur le terroir, et dont les quelques pieds existants (une quarantaine dont 8 à 10 seulement ont 10 à 15 ans d'âge) ont été apportés par le bétail ou plantés par des paysans. 


\subsubsection{Végétation du kori de Séno Ticko et du bas-fond du Diguibari}

Le Kori de Séno Ticko_Il draine tout le bassin versant du même nom. II est surtout réservé aux cultures de

contre-saison. Depuis 1998, les crues, de plus en plus fréquentes du fait des défrichements massifs réalisés sur les glacis qui le bordent, ravagent les cultures céréalières de saison des pluies et les peuplements arborés naturels: Lannea microcarpa, Parkia biglobosa, Tamarindus indica ou cultivés : Mangifera indica.

Le Diguibari_Le bas-fond du Diguibari, large de 200 à $300 \mathrm{~m}$, est inondé pendant les 4 mois de la saison des pluies et conserve des flaques d'eau jusqu'en mars. Même en fin de saison sèche, la nappe phréatique y est peu profonde (< $5 \mathrm{~m}$ ). Les jardins fruitiers, ou les cultures irriguées de saison sèche (piments, oignons, tomates) se développent depuis le début des années quatre vingt dix, avec une accélération de ce mouvement depuis la saison sèche 1997-1998.

Les terrasses et les îlots du bas-fond portent une végétation herbacée caractéristique de ces milieux parmi lesquelles on peut citer les Poaceae : Acroceras amplectens, Chasmopodium caudatum, Echinocloa pyramidalis, Eragrostis spp. (5 espèces), Panicum spp. (5 espèces), Sporobolus indicus var. pyramidalis, Setaria sphacelata, ou les phorbes: Aeschynomene sensitiva, Ipomoea spp. (6 espèces), Melochia corchorifolia, Hygrophylla auriculata, Ludwigia hyssopifolia, Ammania auriculata, etc. Une Borraginaceae prostrée : Coldenia procumbens tapisse les vertisols du lit du Diguibari en saison sèche.

Sur les rives, et particulièrement la rive droite, se développe une belle et haute ripisylve composée en majeure partie par des arbres tels que Mitragyna inermis, Diospyros mespiliformis, Piliostigma reticulatum, Acacia sieberiana, sur lesquels grimpent des espèces lianescentes: Combretum paniculatum, Phyllantus reticulatus, Acacia erythrocalyx, Taccazea apiculata, Ziziphus mucronata, Saba senegalensis. A côté de ces espèces, on trouve des espèces très rares sur le terroir comme Celtis integrifolia ou rares : Vitex doniana, $V$. diversifolia (moins de 15 individus des 2 espèces sur les $6 \mathrm{~km}$ de bas-fond), et des pieds isolés de Ficus gnaphalocarpa, Albizzia chevalieri, Anogeissus leiocarpus, Khaya senegalensis. La population de Khaya senegalensis, composée de grands arbres, est en mauvais état. Elle se partage en de nombreux sujets morts et encore sur pied et d'arbres en train de mourir. II n'a pas été possible de trouver l'origine de ce phénomène : grand âge du peuplement, augmentation de la durée et de la surface couverte par l'inondation annuelle, prélèvement d'écorce à but médicinal trop important, ou association de toutes ces causes?

La partie habituellement inondée du bas-fond est colonisée par un peuplement de Mitragyna inermis adulte, pouvant être très dense par endroits, et par des hydrophytes herbacées : Oryza longistaminata, Pycreus macrostachyos, Pycreus digitatus, Burnatia eneandra, Nymphaea lotus et Utricularia stellaris, dont les organes aériens disparaissent au fur et à mesure du retrait de l'eau.

\section{Discussion}

Les résultats d'étude de la végétation du terroir de Ticko montre que la richesse floristique de ce terroir est supérieure à celle trouvée par Saadou (1994) sur le terroir de Magou, à une vingtaine de kilomètres de Ticko, où 288 espèces et 56 familles ont été répertoriées. Elle est également plus élevée que celle du plateau de Kouré où la forte pression démographique et les sécheresses récurrentes ont induit une dégradation accélérée des formations forestières avec pour conséquence une fragmentation de l'habitat de la girafe (Morou, 2010). Ali et al. (2007) ont également aussi montré au niveau la commune de Gabi située dans la région de Maradi au Niger, que l'avancée du front agricole s'est traduite par une diminution des surfaces couvertes par la savane arbustive plus ou moins arborée.

Les espèces et les familles botaniques d'Angiospermes et Ptéridophytes recensées à Ticko représentent $65 \%$ des espèces et $71 \%$ des familles présentes dans la région du Liptako, qui couvre environ $30000 \mathrm{~km}^{2}$ entre $12^{\circ}$ et $15^{\circ}$ de latitude Nord et $0^{\circ}$ et $3^{\circ}$ de longitude Est (Boudouresque, 1995). Elles représentent 26\% de la flore des Angiospermes du Niger (Saadou, 1990). Par ailleurs, les valeurs du spectre biologique de la flore de Ticko sont proches de celles obtenues par Boudouresque (1995) pour 
le Liptako (Thérophytes : 55\%, Phanérophytes : 22\%) et par Saadou (1990) pour Magou (thérophytes : 57\%, Phanérophytes : 27\%). Aussi le terroir de Ticko peut être considéré comme écologiquement représentatif de la région du Liptako.

Si la richesse floristique à l'échelle du terroir semble avoir peu souffert, pour l'instant, de l'accroissement important des prélèvements et des changements intervenus dans l'occupation des sols, la végétation, elle, a été profondément modifiée sur les glacis, où se trouve la majorité des terres cultivables et des jachères. L'extension des défrichements a fait disparaître les peuplements ligneux originels, dont seuls subsistent quelques arbres reliques. La mise en culture des formations herbacées naturelles, la réduction de la durée et de la surface des jachères, conjuguées à la forte pression de pâture en saison des pluies, ont favorisé l'installation de nouvelles formations végétales. Les formations à grandes Poaceae vivaces ou annuelles (Andropogon gayanus, $A$. pseudapricus, A. fastigiatus, Diheteropogon hagerupii) ont laissé place à des formations herbacées à cycle court (Zornia glochidiata Brachiaria xantholeuca) bien adaptées à la pâture intense mais peu productives, ou à des espèces non consommées par le bétail qui envahissent les surfaces pâturées, comme Sida cordifolia ou Mitracarpus villosus (Hiernaux, 1998 ; Banoin et Achard, 1998 ; Hiernaux et Fernàndez-Rivera, 1999 ; Achard et al., 1999). Ainsi la croissance démographique accompagnée de certains modes d'exploitation entraînent une pression humaine sur les terres avec pour conséquence une perturbation des équilibres environnementaux (Sounon et al., 2007). Selon Kadouza (1996), ce phénomène est particulièrement remarquable en Afrique subsaharienne où les fortes densités démographiques et la crise d'espace agricole des anciens bastions-refuges incitent à l'émigration et à la recherche de terre « vierges ».

Cependant le paysage végétal n'a globalement pas subi de changements majeurs. Les plateaux de Ticko possèdent encore une flore très variée où l'on rencontre des espèces souvent étonnantes dans ce milieu telles que Prosopis africana, Lonchocarpus laxiflorus ou Acacia senegal.
Dans le bas-fond du Diguibari, la mise en place des cultures est relativement récente et ne concerne que la saison sèche. En saison des pluies, l'isolement des îles, les clôtures qui protègent les jardins situés sur la rive droite contre la pâture et la mise en culture de ces surfaces de novembre à avril seulement, permet à la végétation naturelle de boucler son cycle végétatif, et de se régénérer. Dans ce milieu, le système de culture actuel est plutôt favorable à la conservation d'îlots importants de végétation naturelle et à la maintenance de la diversité végétale.

\section{Conclusion}

A Ticko, la diversité floristique est encore à peu près intacte après 25 ans de forte croissance de la densité humaine. On note cependant une érosion des populations d'espèces herbacées et ligneuses, autrefois bien représentées sur le terroir, qui pourrait conduire dans les années à venir à la disparition de ces taxons, et plus particulièrement d'une vingtaine de taxons ligneux. Ce constat pessimiste doit cependant être nuancé : d'une part les disparitions prévisibles ne sont pas certaines, d'autre part, on assiste depuis peu aux premières plantations par les populations d'espèces arborées locales : Prosopis africana, Khaya senegalensis, Adansonia digitata, Parkia biglobosa ou importées : Eucalytus camaldulensis, Prosopis juliflora. Ces plantations sont pour la plupart effectuées à l'intérieur et le long de la clôture de quelques jardins situés sur la terrasse du Diguibari. D'un autre côté, dans le mouvement d'intensification de la production agricole qui se développe depuis une dizaine d'années, on observe une diversification des cultures. Celle-ci se traduit de nos jours par l'importation sur le terroir d'une quinzaine d'espèces légumières ou fruitières.

\section{Références citées}

Achard F., 2001. Végétation du terroir de Ticko (Canton de Torodi - Niger). Inventaire floristique et quelques relevés. IRD - Faculté d'Agronomie, Université Abdou Moumouni de Niamey (Niger), 23 p. + ann. 
Achard F., Ouattara L. \& Banoin M., 1998. Activités de recherche conduites sur le terroir de Ticko, résultats 2000. Projet «Amélioration et gestion de la jachère en Afrique de l'Ouest», Faculté d'Agronomie - ORSTOM, Niamey, Niger, 36p. + ann.

Achard F., Ouattara L. \& Banoin M., 1999. Activités de recherche conduites sur le terroir de Ticko, résultats 1999. Projet «Amélioration et gestion de la jachère en Afrique de l'Ouest», Faculté d'Agronomie - ORSTOM, Niamey, Niger : 41p + ann.

Ali M., Saadou M., Bakasso Y., Abassa I., Aboubacar I. \& Karim S., 2007. Analyse diachronique de l'occupation des terres et caractéristiques de la végétation dans la commune de Gabi (région de Maradi, Niger). Sécheresse 187 (4) : 296-304.

Aubréville A., 1950. Flore forestière soudanoguinéenne. CTFT, Nogent/Marne, France, $523 p$.

Banoin M. \& Achard F., 1998. Place des jachères dans les systèmes d'élevage et comportement alimentaire du bétail (Ticko, sud-ouest nigérien). In : «Jachères et systèmes agraires», eds. C. Floret \& R. Pontanier, communication à l'atelier «jachères et systèmes agraires», Niamey, 30 septembre - 3 octobre 1998, Faculté d'agronomie - ORSTOM, Niger, 99-110.

Berhaut J., 1967. Flore du Sénégal. Clairafrique, Dakar, Sénégal, 485 p.

Boudouresque E., 1995. La végétation aquatique du Liptako (République du Niger). Thèse Doct. es Sciences, université de Paris-Sud, centre d'Orsay, Paris, France. 335p. + ann.

Bouzou M.I., 1998. Evolution de l'occupation du sol dans deux terroirs nigériens: bogodjotou et Ticko. Acte de l'atelier jachère et systèmes agraires, 30 septembre au 2 octobre 1998, Niamey, Niger, pp 15-24.

Gavaud M. \& Boulet R., 1967. Carte pédologique de reconnaissance de la République du Niger (feuille de Niamey). ORSTOM, Paris.

Geerling C., 1982. Guide de terrain des ligneux sahéliens et soudano-guinéens. Wageningen, Neederland, 340p.

Helmstetter D., 1993. Diagnostic régional global de la région de Torodi-Makalondi (canton de
Torodi, Niger). Ministère de l'Hydraulique et de l'Environnement, Niamey, Niger, 62p.

Hiernaux P. \& Fernàndez-Rivera S., 1999. Grazing rotation in the annual-dominated rangelands of the Sahel. In: Eldridge D. and Freudenberger D. (eds), People and Rangelands building the Future, VI International Rangeland Congress, Aitkenvale, Qld, Australia, 513-514.

Hiernaux P. \& Turner M.D., 1996. The effect of clipping on growth and nutrient uptake of sahelian annual rangelands. Journal of Applied Ecology 33: 387-399.

Hiernaux P., 1998. Effects of grazing on plant species composition and spatial distribution in rangelands of the Sahel. Plant Ecology 138: 191-202.

Hutchinson J. \& Dalziel J.M., 1954, Flora of West Tropical Africa, vol. 1, part. 1, 295 p. 1958, vol. 1, part. 2, p. 297-828, vol. 2, 544 p., 1968, vol. 3, part. 1, 276 p. 1972, vol. 3, part. 2, p. 277-574, London, Crown agents for Oversea Governements and Adminitrations, Millbank.

Aboubacar I., 2000. Dynamique et productivité des structures forestières contractées des plateaux de l'ouest nigérien. Thèse Doct., Université Paul Sabatier, Toulouse, France. 216 p. + ann.

Kadouza P., 1996. Colonisation agricole et dynamique de l'espace rural au Togo: étude du cas de la plaine septentrionale du Mono. Mémoire de DEA, FLESH, Université de Lomé, Togo, 81p.

Le Bourgeois Th. \& Merlier H., 1995. Adventrop. Les adventices d'Afrique soudano-sahélienne. CIRAD-CA (ed), Montpellier, France, 640p.

Millington S.J., Tiéga A. and Newby J.E., 1991. Biological Diversity in Niger. WWF, Suisse, 56 p. + ann.

Morou B., 2010. Impacts de l'occupation des sols sur l'habitat de la girafe au Niger et enjeux pour la sauvegarde du dernier troupeau de girafes de l'Afrique de l'Ouest. Thèse de Doctorat Université Abdou Moumouni de Niamey (Niger), 231p.

Ould Mouhamedou O.M. Sidi, 2001. SIG et cartographie de l'occupation du sol en vue de l'aménagement des terroirs: exemple de Ticko et Bogodjotou (Torodi-Niger). Mémoire de fin d'études pour l'obtention du DESS du CRESA (Niamey) 
Peyre de Fabrègues B. \& Lebrun J.P., 1976. Catalogue des plantes vasculaires du Niger. CIRAD-EMVT (ed), Montpellier, France, 433p.

Poilecot P., 1999. Les Poacées du Niger. Description, Illustration, Ecologie, Utilisation. IUCN, CIRAD EMVT. Boissiera 56. Conservatoire et Jardin botanique de la Ville de Genève, Suisse, 766p.

Powell J.M. \& Williams T.O. (1995) An overview of mixed-farming systems in sub-Saharan Africa. In: Powell J.M., Fernàndez-Rivera S., William T.O. and Renard C. (eds), Livestock and Sustainable Nutrient Cycling in Mixed Farming Systems of sub-Saharan Africa. Vol II: Technical Papers, ILCA, Addis Ababa, Ethiopia, 21-36.

RGP (1985) Recensement général de la population de 1977. Résultats définitifs. Rapport d'analyse. Ministère du Plan, Niamey, Niger, 131p. + ann.

RGP (1992) Recensement général de la population 1988. Analyse des données définitives, L'état de la population. Ministère de l'Economie et des Finances, Niamey, Niger, 114p.

Rockström J., 1997. On-farm agrohydrological analysis of the Sahelian yield crisis. Rainfall partitioning, soil nutrients and water use efficiency of pearl millet. Stockholm University, Department of Systems Ecology, Sweden, 3-62.

Saadou M., 1990. La végétation des milieux drainés nigériens à l'est du fleuve Niger. Thèse de Doct. es Sciences, université de Niamey, Niger, 395p. + ann.
Saadou M., 1994. Etude de la flore et de la végétation de la station de Magou. JALDA, Niamey, Niger. Rapp. multigr., 48p.

Saley K., 2001. Etude de la régénération par multiplication végétative naturelle de deux Combrétacées de l'ouest du Niger : Combretum micranthum et Guiera senegalensis. DEA Ecologie Végétale, Université de Ouagadougou, Burkina-Faso, 56p.

Seed-CTFT, 1991. Schéma directeur d'approvisionnement en bois énergie de Niamey. Projet énergie II - énergie domestique. Niamey, Niger, 128p.

Sounon B.B., Sinsin B., \& Goura S. B., 2007. Effets de la dynamique $\mathrm{d}$ »occupation du sol sur la structure et la diversité floristique des forêts claires et savanes du Bénin. Tropicultura 25 (4) 221-227.

Stuart S.N., Adams R.J., \& Jenkins M.D., 1990. Biological Diversity in Sub-saharan Africa and its Islands. Conservation, Management and Sustainable Use. Occasional paper of the IUCN species survival commission $n^{\circ} 6$. IUCN-ICBP, Gland, Switzerland, 145p.

Trochain, J.L., 1957. Accord interafricain sur la définition des types de végétation de l'Afrique Tropicale. Bull. Inst. d'Etudes centrafricaines, nouvelle série, Brazzaville, (n 13-14) 55-93.

Winrock International, 1992. Assessment of animal agriculture in Sub-Saharan Africa. Winrock International Institute for Agricultural Development, Morrilton, Arkansas, USA, 125p. 